\title{
Wahrgenommene Preisfairness bei Revenue Management im Luftverkehr
}

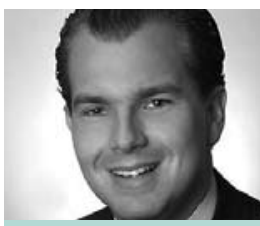

Dipl.-Kfm. Mark Friesen Externer Doktorand am Institut für Öffentliche Dienstleistungen und Tourismus an der Universität St. Gallen $(\mathrm{CH})$

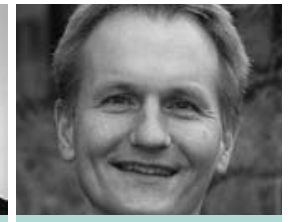

Prof. Dr. Sven Reinecke Dozent an der Universität St. Gallen (HSG) und Leiter des Kompetenzzentrums „Marketing Performance Management" am dortigen Institut für Marketing und Handel, Mitherausgeber von THEXIS (CH)

\section{Wahrgenommene Preis- fairness als Marketingheraus- forderung}

Der Einführung von Revenue Management-Techniken im Luftverkehr werden Erlössteigerungen von durchschnittlich fünf Prozent zugeschrieben. Dem Ziel der kurzfristigen Erlösmaximierung stehen allerdings die - oftmals vernachlässigten potenziell negativen Langfristfolgen durch wahrgenommene Preisunfairness gegenüber. Letztere beeinflusst Einstellung (z. B. Kundenzufriedenheit, Zahlungsbereitschaft) und Kaufverhalten (z. B. Wiederkaufabsicht, Cross-Buying-Aktivität) der Kunden. Im Luftverkehr kommt hinzu, dass der Preis zudem das wichtigste Entscheidungskriterium der Nachfrager ist (Smith et al. 1992, S. 8; Talluri/Van Ryzin 2004, S. 618).

Die wenigen bisherigen Forschungsarbeiten beschränken sich auf die Messung der Preisfairness bei abgeschotteten, kapazitätsbasierten Revenue ManagementSystemen etablierter Fluggesellschaften (Maxwell 2002, S. 489; Wirtz/Kimes 2007, S. 229 ff.). Bisher nicht eindeutig behandelt worden ist die Frage, inwiefern Instrumente des preisbasierten Revenue Managements von Billigfluggesellschaften (Low Cost Carrier) „low-fare no-fair

Revenue Management als eine innovative Form der erlösmaximierenden Preis- und Kapazitätssteuerung rettete in den 80er Jahren eine ganze Branche vor dem Untergang. Mittlerweile fest als Standard im Luftverkehr etabliert, scheint die Frage der wahrgenommenen Preisfairness bei Revenue Management immer noch unbeantwortet. Insbesondere die Flugpreise sogenannter Billigfluggesellschaften eben Anlass zu kundenseitigen Gerechtigkeitsüberlegungen. Der Beitrag analysiert systematisch die unterschiedlichen Revenue Management-Ansätze von etablierten Fluggesellschaften bzw. Billigfluggesellschaften sowie die jeweils vom Kunden empfundene Fairness. Ferner werden Handlungsempfehlungen für ein beziehungsorientiertes Revenue Management abgeleitet.

practices" darstellen (Pena 1998, zitiert nach Seiders/Berry 1998, S. 12). Diese Forschungslücke adressiert der vorliegende Beitrag mittels einer systematischen Gegenüberstellung der beiden Revenue Management-Ansätze und deren wahrgenommener Preisfairness. Ein Transfer der Erkenntnisse auf andere Dienstleistungsbranchen erscheint möglich und sinnvoll, beispielsweise auf das Transportwesen (Schifffahrt, Schienenverkehr, Speditionen) oder das Übernachtungs- und Vermietungsgeschäft (Hotels, Ferienwohnungen, Mietwagenunternehmen).

\section{Kapazitätsbasiertes \\ Revenue Management traditioneller Flug- gesellschaften}

\subsection{Ursprünge des kapazitäts-} basierten Revenue Managements Als Antwort auf die endgültige Deregulierung des US-amerikanischen Luftverkehrs im Jahr 1979 führten die etablierten Fluggesellschaften in den USA eine flexible Preis- und Kapazitätsgestaltung ein. Dieses unter maßgeblicher Beteiligung von American Airlines entwickelte Konzept wird als Revenue bzw. Yield Management bezeich- net. In der deutschsprachigen Literatur wird Revenue Management häufig mit Umsatz- oder Ertragsmanagement, PreisKapazitäts-Steuerung oder Preis-MengenSteuerung übersetzt. Kimes (1989, S. 15) definiert Revenue Management als „[...] process of allocating the right type of capacity to the right kind of customer at the right price so as to maximize revenue or yield“.

\subsection{Instrumente des kapazitäts-} basierten Revenue Managements Abbildung 1 zeigt schematisch den typischen Planungsprozess eines Revenue Management-Systems traditioneller Fluggesellschaften.

Die Entscheidung über die Annahme bzw. Ablehnung unsicherer und zeitlich verteilt eintreffender Nachfrage, d. h. das Problem der Auftragsselektion unter Unsicherheit, lässt sich mathematisch als ein komplexes, nichtlineares Optimierungsmodell beschreiben. Buchungsdatenbanken sowie Planungs- und Prognosemodelle zum zukünftigen Nachfrageverhalten bilden die Informationsgrundlage für den Optimierungsteil des kapazitätsbasierten Revenue Managements. Dieser setzt sich seinerseits aus der Preis- und der Kapazitätssteuerung zusammen. Innerhalb der Preissteuerung werden Nachfrager auf 


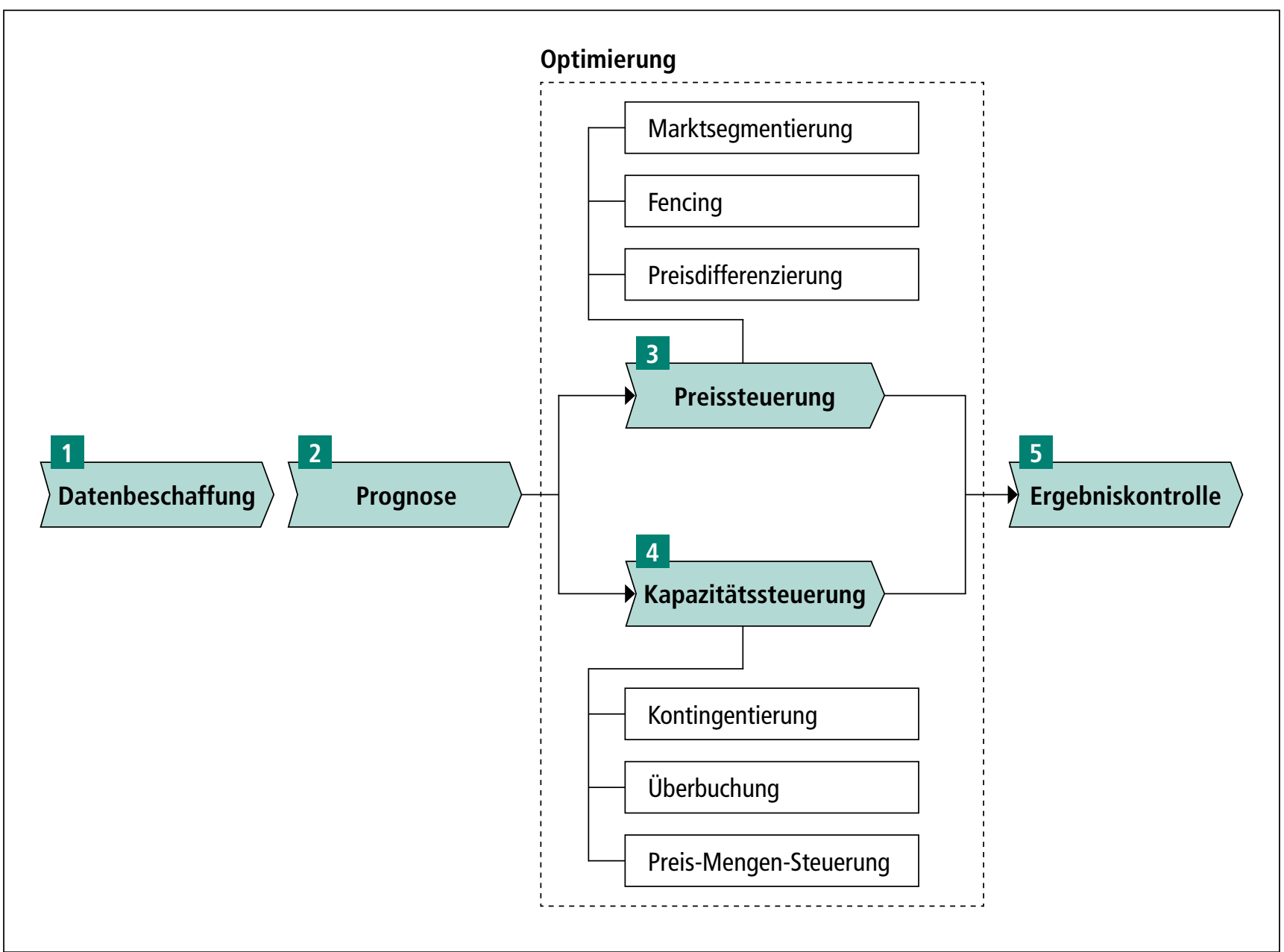

Abb. 1: Vereinfachte Darstellung eines Revenue Management-Planungsprozesses

Quelle: Eigene Darstellung in Anlehnung an Lindenmeier 2005, S. 12.

Grundlage heterogener Zahlungsbereitschaften segmentiert. Auf Basis der identifizierten Marktsegmente werden künstliche Abschottungsregeln definiert, welche die unterschiedlich bepreisten Buchungsklassen innerhalb der Beförderungsklassen (First, Business, Economy Class) voneinander trennen (Lindenmeier 2005, S. 12; Daudel/Vialle 1992, S. 105; Talluri/Van Ryzin 2004, S. 62).

\subsection{Implementierungsformen des kapazitätsbasierten Revenue Managements}

Die unterschiedlichen Zahlungsbereitschaften sind die Grundlage für eine segmentorientierte Preisdifferenzierung, welche die maximale Abschöpfung der Konsumentenrente zum Ziel hat. Gemessen am Grad dieser Abschöpfung findet im Luftverkehr eine Kombination aus Preisdifferenzierungsmaßnahmen zweiten und dritten Grades statt. Für das Revenue Management steht dabei vor allem die zeitliche Preisdifferenzierung im Vordergrund (Simon 1992, S. 42 ff.; Diller 2000, S. 462; Faßnacht 1996, S. 53 ff.). Abbildung 2 zeigt die verschiedenen Implementierungsformen der segmentorientierten Preisdifferenzierung innerhalb des Revenue Managements.

Beim Revenue Management traditioneller Prägung wird die Preisdifferenzierung um die Kapazitätssteuerung ergänzt. Anhand von Nachfrageprognosemodellen werden die beschränkten Kapazitäten in den Buchungsklassen kontingentiert sowie die Buchungslimits bestimmt („Seat-Inventory Control“). Um dem Problem der Ertragswertigkeit der Buchungen operativ zu begegnen, werden zum einen die Kapazitätskontingente geschachtelt („nesting“), zum anderen Kapazitäten bewusst überbucht (Daudel/Vialle 1992, S. 44 und S. 118 ff.; Botimer 1994, S. 70).

Die Preis-Mengen-Steuerung bestimmt Tarifklassen, denen Teilkapazitäten zugeordnet werden. Hierfür stehen statische, dynamische und heuristische Optimierungs- und Planungsmodelle zur Verfügung. Zusätzlich werden die Preise und die dazugehörigen Kapazitäten im Zeitablauf entsprechend der erwarteten Buchungsverläufe angepasst. Dieser Anpassungsvorgang ist bereits Bestandteil der abschließenden Ergebniskontrolle (Daudel/Vialle 1992, S. 53 ff.; Lindenmeier 2005, S. 12 ff.).

\section{Preisbasiertes Revenue Management von Billigflug- gesellschaften}

Obwohl auch dem preisbasierten Revenue Management-Ansatz von Billigfluggesellschaften das Ziel der Erlösmaximierung zu Grunde liegt, unterscheidet sich dieser beträchtlich vom kapazitätsbasierten Ansatz klassischer Fluggesellschaften. Für ein besseres Verständnis wird zunächst die spezielle Preislogik der Low Cost Carrier erläutert. 


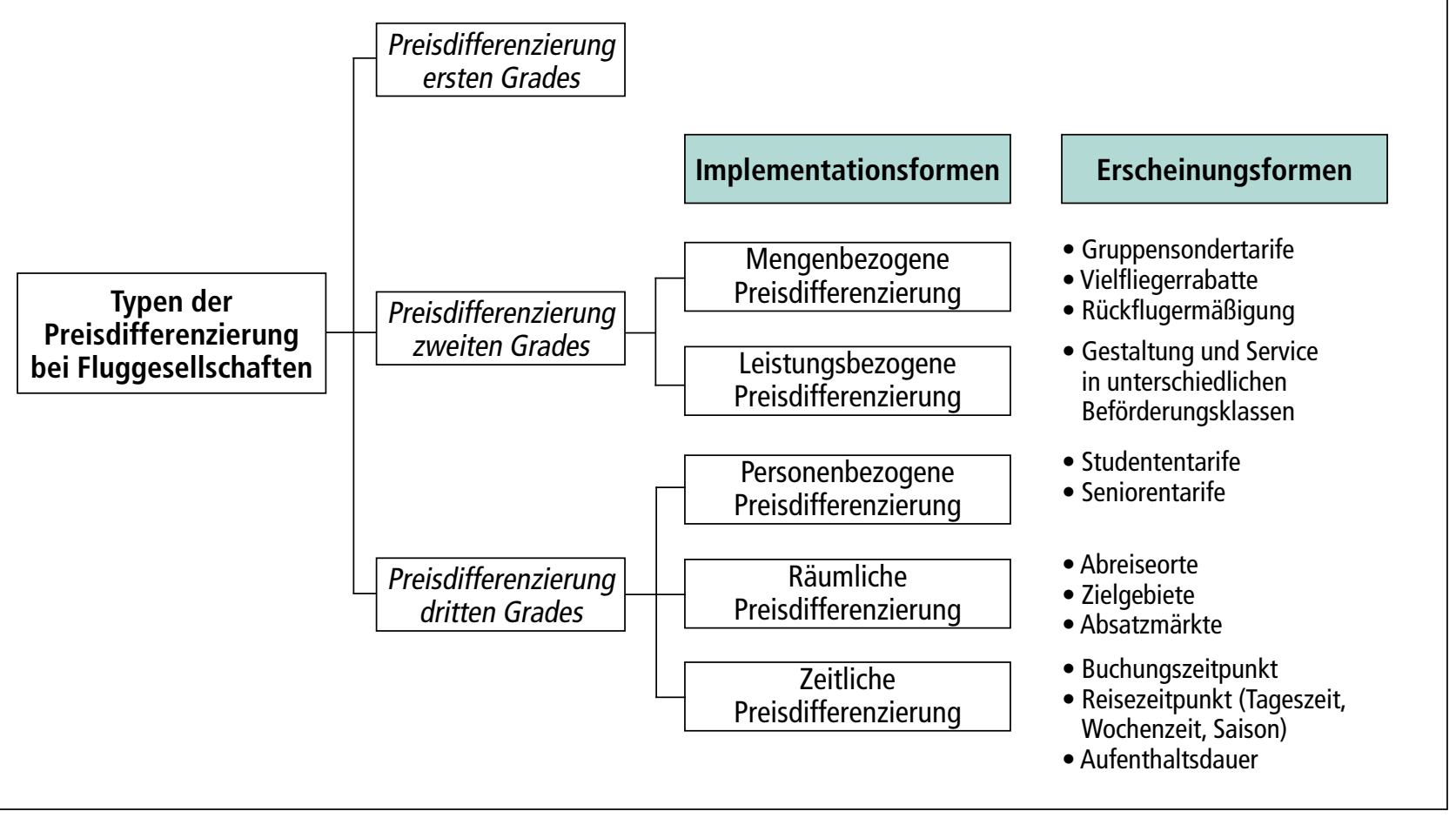

Abb. 2: Typologisierung und Implementierungsformen der Preisdifferenzierung im Luftverkehr

Quelle: Eigene Darstellung in Anlehnung an Pompl 2002, S. 248 ff.

\subsection{Preislogik von Billigflug- gesellschaften}

Die Preislogik der in den USA in den 70er und 80er Jahren (Southwest Airlines und People Express) und in Europa Anfang der 90er Jahre (Ryanair und easyJet) etablierten Billigfluggesellschaften baut darauf auf, dass zu Verkaufsbeginn für einen Flug zuerst die zu unteren Eckpreisen beworbenen Tickets inklusive Steuern und Gebühren (Bruttopreise) verkauft werden, um anschließend die Tarife als Nettopreise progressiv zum Abflugstermin hin zu steigern. Diese Art der schrittweisen Preiskurve bietet dem Kunden die Garantie, dass im späteren Verlauf keine günstigeren Tickets mehr angeboten werden. Die zentrale Innovation neben dem gesenkten Preisniveau und der simplifizierten Preisstruktur besteht in der Verfügbarkeit von Flugtickets auf EinwegBasis mit gar keinen oder nur minimalen Restriktionen. Daher wird dem Kunden bei seiner Buchungsanfrage zu einem bestimmten Zeitpunkt stets immer nur ein Preis für einen Sitzplatz auf einem bestimmten Flug angezeigt (Friesen 2006, S. 104).

\subsection{Instrumente des preisbasierten Revenue Managements}

Billigfluggesellschaften setzen somit den Preis als alleinige Optimierungs- und
Steuerungsvariable ein. Im Rahmen des Optimierungsteils kombinieren Low Cost Carrier Elemente der konventionellen Preis- und Kapazitätsteuerungsinstrumente traditioneller Fluggesellschaften. Eine explizite Marktsegmentierung findet beim preisbasierten Revenue Management allerdings nicht statt. Stattdessen werden Nachfrager hauptsächlich über den Zeitpunkt der Buchung und der Inanspruchnahme segmentiert. Annahmegemäß unterstellt die Anwendung zeitlicher Preisdifferenzierung, dass kurzfristige Nachfrage weniger zeitlich flexibel ist und deshalb über eine höhere Zahlungsbereitschaft verfügt (Burger/Fuchs 2004, S. 40).

Während einige Billigfluggesellschaften Buchungskorridore vorgeben und die Preise bei Über- oder Unterschreiten entsprechend verändern, haben andere wiederum teil- oder vollautomatische Planungs- und Optimierungsmodelle entwickelt. Diese erlauben es, auf Basis geschätzter, durchschnittlicher Preis- und Kreuzpreiselastizitäten in verschiedenen Zeitintervallen auf der Buchungskurve einen erlösoptimalen Preis zu bestimmen.

Die Preissteuerung auf Basis geschätzter Preiselastizitäten in Verbindung mit nichtphysischen „Fences“ reicht aus, um die Nachfrage erlösoptimal zu allozieren. Eine
Kontingentierung beschränkter Kapazitäten ist bis auf wenige Ausnahmen (Werbeeckpreise, Preisaktionen) nicht notwendig und lässt eine aktive Preis-MengenSteuerung obsolet werden. Billigfluggesellschaften machen sich lediglich den bewussten Überverkauf von Kapazitäten durch Überbuchung zur Erlösmaximierung zu eigen.

\subsection{Vergleich von kapazitäts- und preisbasiertem Revenue Management}

Der grundlegende Unterschied zwischen dem Revenue Management von Billigfluggesellschaften und jenem etablierter Netzwerkfluggesellschaften liegt darin, dass bei Letzterem eindeutig die Steuerung der Kapazitäten und nicht die der Preise im Vordergrund steht (Abb. 3). Preisänderungen sind die Folge der Steuerung von Verfügbarkeiten unterschiedlich bepreister Sitzplatzkapazitäten in mehreren $\mathrm{Bu}$ chungsklassen. Im Gegensatz dazu versuchen Billigfluggesellschaften ihre Preise dynamisch einer vorher festgesetzten Kapazität erlösmaximierend anzupassen (Daudel/Vialle 1992, S. 124; Faßnacht 1996, S. 92).

Aufgrund des zunehmenden Wettbewerbs haben sich in der jüngeren Vergan- 
genheit die dichotomen Revenue Management-Ansätze allerdings angenähert, wie die „betterFly“-Angebote von Lufthansa und die Flex-Tarife von Germanwings oder Air Berlin zeigen.

\section{Wahrgenommene Preisfair- ness bei Revenue Management im Luftverkehr}

Trotz der Hybridisierung der beiden Revenue Management-Modelle sind die in der verhaltenswissenschaftlichen Preistheorie verorteten, kognitiven Konsumentenreaktionen auf die unterschiedlichen Ansätze voneinander zu trennen.

\subsection{Distributive Ungerechtigkeit von Revenue Management}

Die Akzeptanz traditioneller, kapazitätsbasierter Revenue Management-Ansätze war insbesondere nach der Einführung bei US-amerikanischen Linienfluggesellschaften Ende der 70er Jahre gering. Immer stärker ausdifferenzierte Tarifstrukturen mit tausenden täglicher Tarifwechsel als Folge von Preisdifferenzierungsinstrumenten zweiten und dritten Grades sorgten für Verwirrung und Verärgerung unter den Fluggästen. Eine europäische Airline beispielsweise arbeitet heute noch mit bis zu 1,5 Millionen verschiedenen Tarifen weltweit, die sich 10.000 Mal täglich än- dern können. Dass der gleiche Sitzplatz in einem Flugzeug in Abhängigkeit von z. B. geographischen oder zeitlichen Faktoren $\mathrm{zu}$ unterschiedlichen Preisen angeboten wurde, löste Bedenken bezüglich der distributiven Gerechtigkeit der Preishöhe bei Konsumenten aus (Botimer 1994, S. 13).

Im Gegensatz dazu ist das preisbasierte Revenue Management von Billigfluggesellschaften insbesondere durch die Preisdiskriminierung einer homogenen Kernleistung bei Fehlen physischer Preisbarrieren gekennzeichnet. Dadurch entsteht Preisfairness exklusiv auf Basis eines Preisvergleichs. Die Gefahr, dass die Preisdifferenzierungsmaßnahmen dritten Grades als tendenziell unfair wahrgenommen werden, ist durch die Gleichrangigkeit der Passagiere weitaus größer. Kimes (2002, S. 21) bestätigt, dass: „A customer who pays more for a similar service and cannot perceive a difference in the service may view the situation as unfair."

\subsection{Verfahrensungerechtigkeit von Revenue Management}

Dynamische Preissetzungsverfahren wie das Revenue Management, welche sich nach den Marktkräften richten, werden in der Theorie der Verfahrensgerechtigkeit von Kunden als grundsätzlich unfair wahrgenommen. Dies gilt vor allem für die Kapazitätssteuerung im Rahmen des Revenue Managements traditioneller Fluggesell- schaften. Werden Flüge überbucht oder Kapazitäten für eine bestimmte höherwertige Kundenklientel freigehalten bzw. Discount-Tarife kurz vor Abflug geschlossen, empfinden die benachteiligten Nachfragersegmente dies als unfair (Campbell 1999, S. 147; Wirtz et al. 2003, S. 223).

Reaktanzen gegenüber dem Preissetzungsverfahren von Billigfluggesellschaften ergeben sich aus der Unsicherheit der Nachfrager über den Preisverlauf, aus der Nichtverfügbarkeit von Kapazitäten und der wahlweisen Preisdarstellung als Bruttooder Nettopreis. Nicht zu vergessen sind die in zahlreichen Preisaktionen aggressiv beworbenen Promotionspreise, die kapazitätsbeschränkt nur für einen bestimmten Zeitraum buchbar sind. Dabei besteht die Gefahr, dass Kunden ihren als fair empfundenen Referenzpreis an den günstigen Aktionspreis koppeln. Erhalten sie diesen bei ihrer nächsten Buchung nicht, nehmen sie das Preissetzungsverfahren als unfair wahr (Huang et al. 2005, 358 ff.).

\subsection{Fairness von Revenue Management}

Aus den Forschungsarbeiten zum Dual Entitlement-Prinzip von Kahneman et al. (1986a, 1986b) ist bekannt, dass Nachfrager Preisunterschiede dann als fair wahrnehmen, wenn diese durch zurechenbare Kostendifferenzen gerechtfertigt sind. Aufgrund dessen konnten diskriminie-

\begin{tabular}{|c|c|}
\hline $\begin{array}{c}\text { Revenue Management von } \\
\text { traditionellen Fluggesellschaften }\end{array}$ & $\begin{array}{l}\text { Revenue Management von } \\
\text { Billigfluggesellschaften }\end{array}$ \\
\hline $\begin{array}{l}\text { - Produktdifferenzierung } \\
\text { - Hauptsächlich Retour-Tarife } \\
\text { - Verfügbarkeit von günstigeren Tarifklassen nimmt mit } \\
\text { - Näherrücken des Abflugdatums ab } \\
\text { - Tarifdifferenzierung gemäß Produktdifferenzierung } \\
\text { - Vielzahl von Buchungsklassen zu einem Zeitpunkt } \\
\text { - Physisegmentierung über Tarifabschottungen } \\
\text { - Preissteuerung über heterogene Zahlungsbereitschaften } \\
\text { und Abschottungsregeln } \\
\text { - Buchungskontrolle über Verfügbarkeit von Kapazitäten } \\
\text { - Protektionsmechanismen von höherwertiger Nachfrage } \\
\text { (Nesting) } \\
\text { - Preis-Mengen-Steuerung } \\
\text { - Aptimaler Tarif-Mix } \\
\text { gesellschaften über „betterFly“-Angebote (Lufthansa) }\end{array}$ & $\begin{array}{l}\text { - Keine Produktdifferenzierung } \\
\text { - Ausschließlich One-Way-Preise } \\
\text { - Grundsätzlicher Preisanstieg bis zum Abflugszeitpunkt } \\
\text { - Ein Produkt zu verschiedenen Preisen } \\
\text { - Nur ein Preis zu einem bestimmten Zeitpunkt } \\
\text { - Keine Marktsegmentierung } \\
\text { - Lediglich nicht-physische Tarifabschottungen } \\
\text { - Preissteuerung über Buchungskorridore oder } \\
\text { - Preiselastizitäten } \\
\text { - Buchungskontrolle über angebotenen Preis } \\
\text { - Keine Protektionsmechanismen für höherwertige } \\
\text { Nachfrage } \\
\text { - Keine Preis-Mengen-Steuerung erforderlich } \\
\text { - Optimale Kontrolle der hohen Preise kurz vor Abflug } \\
\text { - Annäherung an Revenue Management von traditionellen } \\
\text { Fluggesellschaften über Flex-Tarife (Germanwings und Air } \\
\text { Berlin) }\end{array}$ \\
\hline Kapazitätsbasiert & Preisbasiert \\
\hline
\end{tabular}

Abb. 3: Vergleich von kapazitäts- und preisbasiertem Revenue Management 
rende Tarifunterschiede bei traditionellen Fluggesellschaften mitunter noch durch entsprechende Kostenunterschiede in den unterschiedlichen Beförderungsklassen kommunikativ begründet werden (Vaidyanathan/Aggarwal 2003, S. 462; Reinecke/ Hahn 2003, S. 345).

Wirkungsvoller jedoch als die rein kommunikative Aufklärung der Konsumenten ist für die Fairness-Wahrnehmung von kapazitäts- und preisbasierten Revenue Management-Praktiken der Einsatz sogenannter Tarifabschottungen („Rate Fences“). Unter „Fences“ sind physische und nichtphysische Barrieren zwischen den Preissegmenten zu verstehen, welche Airlines eine belastbare Begründung liefern, um Kunden unterschiedlich hohe Preise anzubieten (Abb. 4) (Wirtz/Kimes 2007, S. 230; Schwietermann 1985, S. 33 ff.).

Während traditionelle Fluggesellschaften gleichzeitig auf den Einsatz physischer und nicht-physischer Buchungsregeln und -restriktionen zurückgreifen, kommen bei
Billigfluggesellschaften aufgrund der Homogenität der Transportleistung ausschließlich nicht-physische Preisbarrieren zum Einsatz. In beiden Fällen erlauben es die Abschottungsregeln den Konsumenten, sich gemäß ihrer individuellen Zahlungsbereitschaft und Zeitsensitivität frei und eigenständig zu segmentieren. Dies liefert Nachfragern eine Grundlage dafür, die sowohl durch das kapazitäts- als auch das preisbasierte Revenue Management erzeugten, unterschiedlich abgeschotteten Flugpreise als fair wahrzunehmen (Kimes 2002, S. 24 ff.; Friesen 2006, S. 105 ff.).

Nicht nur der wirkungsvolle Einsatz von Preisabschottungen durch etablierte Airlines und Billigfluggesellschaften hat dazu beigetragen, Revenue Management als eine in den Augen der Konsumenten akzeptable Preissetzungsregel zu etablieren. Vielmehr hat auch die bloße Vertrautheit der Nachfrager mit Revenue Management-Techniken geholfen, anfängliche Unfairness-Urteile ins Gegenteil zu verkehren.
Insbesondere für das Revenue Management etablierter Airlines gilt, dass sich Luftverkehrsnachfrager aufgrund ihrer jahrelangen, täglichen Erfahrungen schlichtweg an die jeweiligen Preissysteme gewöhnt haben. Dass diejenigen Verfahren, die Nachfrager häufiger antreffen, letztlich auch als fairer empfunden werden, ist intuitiv einleuchtend und wird sowohl von der Gerechtigkeitstheorie als auch der Normtheorie bestätigt (Talluri/Van Ryzin 2004, S. 616 f.; Kimes 2002, S. 21 f.; Bieger 1998, S. 280).

Dieses gilt partiell auch für die Preispolitik von Billigfluggesellschaften. Den heutigen Low Cost-Passagieren kann unterstellt werden, dass sie das Preissystem größtenteils internalisiert und akzeptiert haben. Vor allem, dass jeder Nachfrager auf Basis einer „first come, first served“Regel dieselbe Chance hat, ein günstiges Low Cost-Flugticket zu erwerben, lässt das Preissystem der Billigfluggesellschaften als relativ fair erscheinen (Talluri/Van Ryzin 2004, S. 616 f.; Calder 2002, S. 197 ff.).

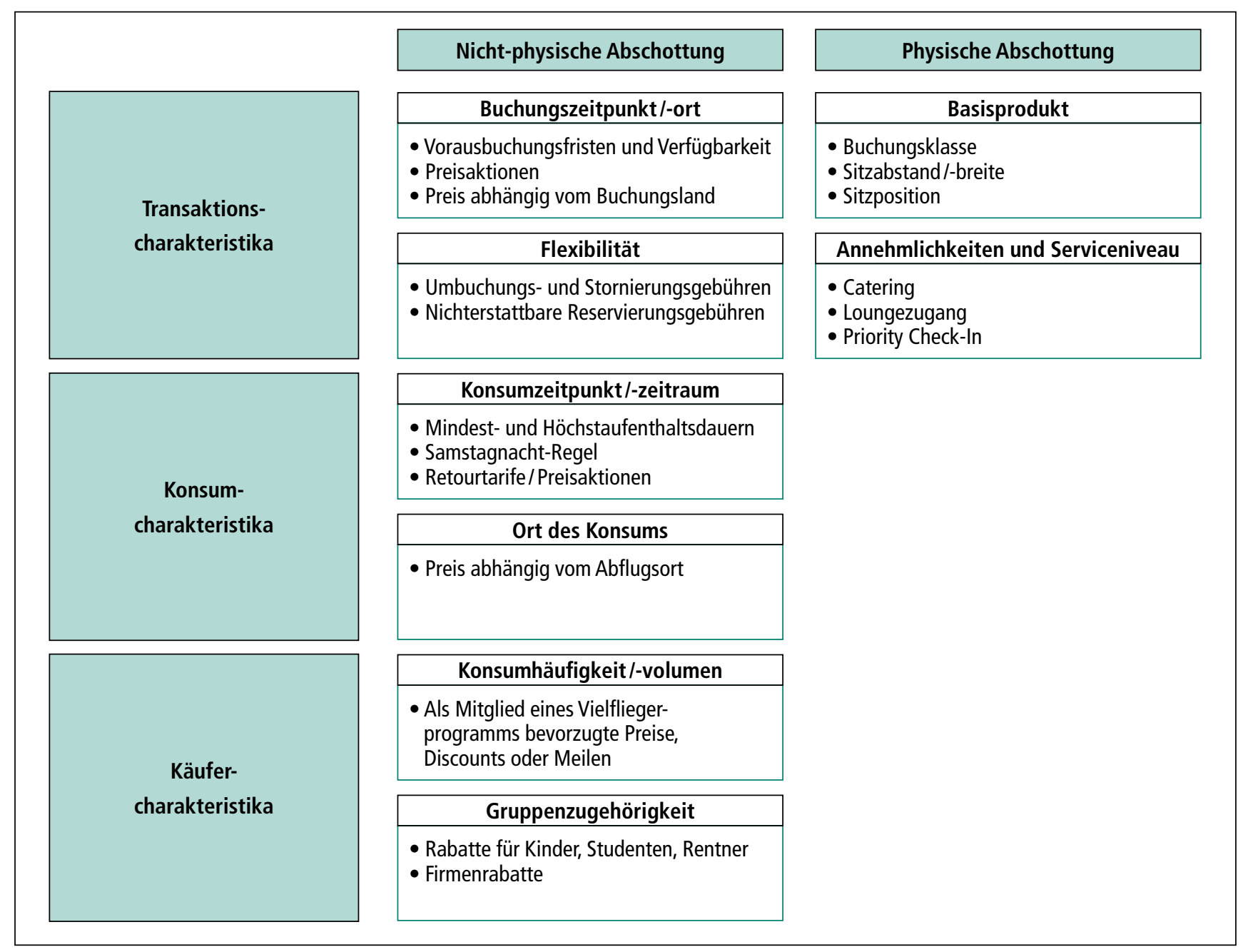

Abb. 4: Verschiedene Arten der Tarifabschottung im Luftverkehr 


\section{Fazit und Handlungs- empfehlungen}

Ausgangspunkt des vorliegenden Beitrags war die Frage, inwiefern Revenue Management von etablierten Airlines und von Billigfluggesellschaften seitens der Nachfrager als fair bzw. unfair wahrgenommen wird. Es konnte in beiden Fällen gezeigt werden, dass Kunden nicht die grundsätzliche Logik des Revenue Managements per se als unfair einschätzten, sondern vereinzelt die operative Umsetzung im Luftverkehr. Damit liegt die wirkliche Herausforderung in der Unternehmenspraxis darin, ein erlösoptimales Revenue Management zu implementieren, dessen Instrumente gleichzeitig von den Konsumenten auch als fair und akzeptabel wahrgenommen werden. Hierzu bieten sich insbesondere die folgenden drei Handlungsempfehlungen für ein kapazitäts- wie auch preisbasiertes Revenue Management an:

(1) Kommunikation intensivieren

Die aktive Information und Offenlegung und Kommunikation der Logik des Preissetzungsverfahrens hilft, die wahrgenommene Preisfairness von Revenue Management-Praktiken zu verbessern, denn: „No fairness effort will work without communication." (Seiders/Berry 1998, S. 17). Gerade Neukunden von Billigfluggesellschaften empfinden es als unfair, wenn die Information fehlt, dass der Preis zum Abflug hin grundsätzlich ansteigt.

(2) Tarifabschottungen setzen

Neben kommunikativen Maßnahmen können physische und nicht-physische Abschottungsregeln höchst erfolgreich sein, um Konsumenten eine Selbstselektion auf Basis ihrer Zahlungsbereitschaft und Zeitsensitivität zu ermöglichen. Das willkürliche Setzen von Tarifbestimmungen allein reicht jedoch nicht aus. Vielmehr müssen „Fences“ logisch, eindeutig und schwer zu umgehen sein, um als fair wahrgenommen zu werden (Wirtz et al. 2003, S. 22).

\section{(3) Preisanstiege erklären}

Basierend auf den Erkenntnissen des Dual Entitlement-Prinzips von Kahneman et al. (1986a, 1986b) steigert eine exakte Erklärung der Ratio hinter einem Preisanstieg den Fairness-Charakter von Revenue Management. Idealerweise kann eine Preiserhöhung mit externen Faktoren, die außerhalb des Einflussbe- reichs des Unternehmens liegen, begründet werden, wie z. B. gestiegenen Treibstoffkosten (Talluri/Van Ryzin 2004, S. 618 f.).

\section{Literatur}

Bieger, T. (1998): Dienstleistungsmanagement: Einführung in Strategien und Prozesse bei persönlichen Dienstleistungen mit Fallstudien verschiedener Praktiker, Bern.

Botimer, T. C. (1994): Airline Pricing and Fare Product Differentiation, Dissertation, Cambridge.

Burger, B./Fuchs, M. (2004): Dynamic pricing - A future airline business model, in: Journal of Revenue and Pricing Management, Vol. 4, No. 1, S. 39-53.

Calder, S. (2002): No frills: The truth behind the low-cost revolution in the skies, London.

Campbell, M. C. (1999): „Why did you do that?" The important role of inferred motive in perceptions of price fairness, in: Journal of Product \& Brand Management, Vol. 8, No. 2, S. 145-152.

Cox, J. L. (2001): Can differential prices be fair?, in: Journal of Product \& Brand Management, Vol. 10, No. 5, S. 264 275.

Daudel, S./Vialle, G. (1992): Yield Management: Erträge optimieren durch nachfrageorientierte Angebotssteuerung, Frankfurt/New York.

Diller, H. (2000): Preispolitik, Stuttgart/ Berlin/Köln.

Fassnacht, M. (1996): Preisdifferenzierung bei Dienstleistungen: Implementationsformen und Determinanten, Wiesbaden.

Friesen, M. (2006): Die Preiswahrnehmung bei europäischen Low Cost Airlines - Eine phänomenologisch-explorative Untersuchung anhand von Fokusgruppen, in: Internationales Verkehrswesen, 58. Jg., Nr. 3, S. 104-107.

Huang, J.-H./Chang, C.-T./Chen, C. Y.-H. (2005): Perceived fairness of pricing on the Internet, in: Journal of Economic Psychology, Vol. 26, No. 3, S. 343-361.

Kahneman, D./Knetsch, J. L./Thaler, R. H. (1986a): Fairness as a Constraint on Profit Seeking: Entitlements in the Market, in: The American Economic Review, Vol. 76, No. 4, S. 728-741.
Kahneman, D./Knetsch, J. L./Thaler, R. H. (1986b): Fairness and the Assumptions of Economics, in: Journal of Business, Vol. 59, No. 4, S. 285-300.

Kimes, S. E. (1989): The Basics of Yield Management, in: Cornell Hotel \& Restaurant Administration Quarterly, Vol. 30, No. 3, S. 14-19.

Kimes, S. E. (2002): Perceived Fairness of Yield Management, in: Cornell Hotel \& Restaurant Administration Quarterly, Vol. 43, No. 1, S. 21-30.

Lindenmeier, J. (2005): Yield-Management und Kundenzufriedenheit: Konzeptionelle Aspekte und empirische Analyse am Beispiel von Fluggesellschaften, Wiesbaden.

Maxwell, S. (2002): Rule-based price fairness and its effect on willingness to purchase, in: Journal of Economic Psychology, Vol. 23, No. 2, S. 191-212.

Reinecke, S./Hahn, S. (2003): Preisplanung, in: Diller, H./Herrmann, A. (Hrsg.): Handbuch Preispolitik, Wiesbaden, S. 333-355.

Schwietermann, J. P. (1985): Fare is Fair in Airline Deregulation: The Decline of Price Discrimination, in: Regulation, Vol. 14, No. 3, S. 32-38.

Seiders, K./Berry, L. L. (1998): Service fairness: What it is and why it matters, in: Academy of Management Executive, Vol. 12, No. 2, S. 8-20.

Simon, H. (1992): Preismanagement, Wiesbaden.

Smith, B. C./Leimkuhler, J. F./Darrow, R. $M$. (1992): Yield Management at American Airlines, in: Interfaces, Vol. 22, No. 1, S. 8-31.

Talluri, K. T./Van Ryzin, G. J. (2004): The Theory and Practice of Revenue Management, Boston et al.

Vaidyanathan, R./Aggarwal, P. (2003): Who is the fairest of them all? An attributional approach to price fairness perceptions, in: Journal of Business Research, Vol. 56, No. 6, S. 453-463.

Wirtz, J./Kimes, S. E. (2007): The Moderating Role of Familiarity in Fairness Perceptions of Revenue Management Pricing, in: Journal of Service Research, Vol. 9, No. 3, S. 229-240.

Witz, J./Kimes, S. E./Theng, J./Patterson, P. (2003): Revenue management: Resolving potential customer conflicts, in: Journal of Revenue and Pricing Management, Vol. 2, No. 3, S. 216-226. Х 